\title{
Association between gestational diabetes and perinatal depressive symptoms: evidence from a Greek cohort study
}

\author{
Pinelopi Varela ${ }^{1}$, Areti C. Spyropoulou ${ }^{1}$, Zacharias Kalogerakis ${ }^{1}$, Eleni Vousoura ${ }^{1}$, Martha Moraitou ${ }^{2}$ and \\ lannis M. Zervas ${ }^{1}$ \\ ${ }^{1}$ First Department of Psychiatry, Women's Mental Health Clinic, Athens University Medical School, Athens, Greece \\ ${ }^{2}$ Department of Midwifery, Alexander Technological Educational Institute, Thessaloniki, Greece
}

\begin{abstract}
Aim: The aim of the present study was to assess the association of gestational diabetes mellitus (GDM) with prenatal and postnatal depressive symptoms in a sample of pregnant women in Greece. Background: Earlier research supports a relationship between depression and diabetes, but only a few studies have examined the relationship between GDM and perinatal depressive symptomatology. Methods: A total of 117 women in their third trimester of pregnancy participated in the study. Demographic and obstetric history data were recorded during women's third trimester of pregnancy. Depressive symptoms were assessed with the validated Greek version of the Edinburg Postnatal Depression Scale (EPDS) at two time points: on the third trimester of pregnancy and on the first week postpartum. Findings: Prevalence of GDM was $14.5 \%$. Probable diagnosis of depression occurred for $12 \%$ of the sample during the antenatal assessment and $15.1 \%$ in the postpartum assessment. In the first week postpartum, women with GDM had significantly higher postpartum (but no antenatal) EPDS scores compared with the non-GDM cohort. In conclusion, GDM appears to be associated with depressive symptoms in the first week postpartum. Clinical implications and recommendations for future research are discussed, emphasizing the importance of closely monitoring women with GDM who seem more vulnerable to developing depressive symptomatology during the postnatal period.
\end{abstract}

Key words: gestational diabetes mellitus (GDM); maternal mental health; perinatal depression

Received 9 November 2016; revised 10 April 2017; accepted 7 May 2017;

first published online 5 June 2017

\section{Introduction}

Gestational diabetes mellitus (GDM) is defined as any degree of glucose or carbohydrate intolerance with onset or first recognition during pregnancy (ACOG Committee on Practice Bulletins-Obstetrics, 2004; International Association of Diabetes and Pregnancy Study Groups Consensus Panel et al., 2010). GDM is among the most common medical disorders of the perinatal period affecting up to $21 \%$ of women globally (Bener et al., 2011). A current

Correspondence to: Pinelopi Varela, Vas. Sofias 74, 11528, Athens, Greece. Email: pinelopimid@yahoo.gr study estimated that the international prevalence of hyperglycemia in pregnancy in women (20-49 years) is $16.9 \%$, or 21.4 million live births in 2013 (Guariguata et al., 2014). With an everincreasing prevalence worldwide, GDM constitutes a major public health concern considering its adverse effects on maternal and neonatal outcomes (Crowther et al., 2005; Metzger, 2007).

GDM is a well-known risk factor for developing type 2 diabetes in the future. Women with a history of GDM have a sevenfold increased risk of developing type 2 diabetes within 10 years of the affected pregnancy (Bellamy et al., 2009). These women also have an increased risk for cardiovascular diseases. 
Moreover, GDM is a well-established risk factor for a wide range of complications during pregnancy and birth, to include pre-eclampsia, hypertension, cesarean section, and preterm birth (Metzger, 2007). Importantly, infants of mothers with GDM have several short- and long-term adverse outcomes including macrosomia, preterm birth, metabolic disorders such as hypoglycemia, hypocalcemia, hyperbilirubinemia, respiratory disorders, obesity, and cognitive impairments (Crowther et al., 2005; Metzger, 2007; Mitanchez et al., 2015).

Another common condition of the perinatal period is depression. Between 14 and $23 \%$ of pregnant women will experience a depressive disorder during pregnancy (Gaynes et al., 2005) and 15-20\% of women will develop postpartum depression (PPD) after giving birth (Davé et al., 2010). A latest review reported its prevalence to be $1.9-82.1 \%$ and $5.2-74.0 \%$ in developing and developed countries, respectively, using a self-reported questionnaire. Its prevalence has also been reported to vary from 0.1 to $26.3 \%$ using a structured clinical interview (Norhayati et al., 2015). Perinatal depression has detrimental consequences for the lives of women, children, and their families. Prenatal depressive symptomatology has been linked to poor maternal self-care and outcomes. Pregnant women who experience depressive symptoms are less likely to look for proper medical care during pregnancy (Leung and Kaplan, 2009). Untreated PPD can have long-lasting negative effects on the woman, the maternal role, the mother-child relationship, child development, and the marital relationship (Murray et al., 1996; Weinberg and Tronick, 1998; Field, 2010).

There is evidence of a bidirectional relationship between depression and diabetes. Depression earlier in life increases the risk for development of type 2 diabetes (Golden et al., 2008) and diabetesspecific complications are associated with a higher risk of subsequent depression (Katon et al., 2009). Diabetes doubles the likelihood of comorbid depression, which is present in $\sim 30 \%$ of patients with type 1 or type 2 diabetes (de Groot et al., 2001).

Less is known about the relationship between GDM and perinatal depressive symptoms. Previous studies have identified an association between GDM and prenatal depressive symptoms (Kozhimannil et al., 2009); however, contradicting findings have also been reported (Katon et al., 2011; Byrn and Penckofer, 2015). Moreover, a number of studies indicate an association between GDM and PPD
(Dalfrà et al., 2001), while others have not confirmed this association (Kim et al., 2005; Besser et al., 2007; Halkoaho et al., 2010; Al-Shahrani et al., 2011; Liu and Tronick, 2013).

\section{Purpose}

The main aim of this study is to examine the association between GDM with prenatal depressive symptoms in the third trimester of pregnancy and PPD symptoms in the first week postpartum, in a sample of pregnant women in Greece. To the authors' knowledge, no similar research has been conducted in Greece.

\section{Methods}

\section{Sample}

Participants were recruited from the practice of collaborating obstetricians in Athens, Greece. The study sample consisted of a total of 117 women on their third trimester of pregnancy who received regular prenatal care. Eligible participants were women in their third trimester of pregnancy of Greek origin or fluent in Greek language, who were provided with a detailed description of the study procedures and signed a written informed consent before the inclusion in the study. Exclusion criteria were active psychotic symptoms, organic brain pathology, and intellectual disability. The study protocol was approved by the assembly of Athens University Medical School (approval number: 6761) for scientific and ethical standards.

\section{Measures}

Data were collected at two time points: (1) during the third trimester (anytime between 32nd and 35th week) of pregnancy; and (2) within the first week postpartum. Demographic and general health information were assessed during the prenatal assessment, while the Edinburgh Postnatal Depression Scale (EPDS) (Cox et al., 1987) and obstetric information at both time points. Participants were asked to complete a package of self-report measures including socio-demographic information, a detailed obstetric history, and current health status.

Prenatal depressive and PPD symptoms were assessed with the EPDS. The EPDS is a self-report questionnaire assessing the severity of depression 
symptoms during pregnancy and postpartum. It consists of 10 items, each rated on a four-point scale; items three and five through 10 are reverse scored. Range of possible score is between 0 and 30, and higher scores suggest greater severity of depressive symptoms. EPDS is a widely used screening tool for perinatal depression with satisfactory psychometric properties both in prenatal and postnatal populations (Cox et al., 1987). The Greek version of EPDS has been validated for perinatal use (Leonardoua et al., 2009). For the purposes of this study, diagnosis of probable major depression was set at the wellvalidated cut-off point of 12/13 (Cox et al., 1987).

\section{Data analysis}

Descriptive statistics were calculated for initial data analysis and demographic, clinical, and obstetric data were compared using Student's $t$, MannWhitney $U, \chi^{2}$, or Fisher's exact tests, as appropriate. Differences in mean EPDS scores between GDM and non-GDM groups were explored both during pregnancy and postpartum. Because EPDS scores were non-normally distributed as evidenced by plotted data and significant Kolmogorov-Smirnov normality test $(P<0.001)$, non-parametric tests were used (Mann-Whitney $U$ ). Logistic regression analyses were also conducted, with EPDS score as a categorical dependent variable (probable major depressive disorder (MDD) diagnosis $\geqslant 13$ versus no MDD diagnosis $\leqslant 12$ ). We first tested models including pregnancy or postpartum probable MDD diagnosis only in the model, and then models controlling for confounding variables (age, nulliparity, mode of delivery, and neonatal intensive care unit (NICU) admission). Statistical significance level was set at $P$-value of 0.05 . Data management and analysis were performed using SPSS software package version 21.0.

\section{Findings}

Table 1 presents the demographic and clinical characteristics of the sample. In brief, the average age of women was $32.6(\mathrm{SD}=4.06)$ years. The majority was married $(95.7 \%)$ and predominantly Greek (93.2\%), and had completed higher education $(65.0 \%)$. In addition, $83.8 \%$ of them were employed, with most of them $(56.4 \%)$ having a monthly family income between $€ 1000$ and 3000 .

Table 1 Descriptive statistics of the original $(n=117)$ and follow-up $(n=93)$ sample

\begin{tabular}{|c|c|c|c|c|}
\hline & Original & Follow-up & Drop out & \\
\hline Married & $112(95.7)$ & $89(95.7)$ & $23(95.8)$ & n.s. \\
\hline \multicolumn{4}{|l|}{ Educational level } & n.s. \\
\hline$<$ High school diploma & $3(2.6)$ & $1(1.1)$ & $2(8.3)$ & \\
\hline High school diploma & $38(32.5)$ & $30(32.3)$ & $8(33.3)$ & \\
\hline Bachelor's degree & $76(65.0)$ & $62(66.7)$ & $14(58.3)$ & \\
\hline \multicolumn{4}{|l|}{ Professional status } & n.s. \\
\hline Employed & $98(83.8)$ & $81(87.1)$ & $17(70.8)$ & \\
\hline Unemployed & $7(6.0)$ & $5(5.4)$ & $2(8.3)$ & \\
\hline Housemaker & $12(10.3)$ & $7(7.5)$ & $5(20.8)$ & \\
\hline Greek nationality & 109 (93.2) & $91(97.8)$ & $18(75.0)$ & $P<0.001$ \\
\hline \multicolumn{5}{|l|}{ Monthly family income } \\
\hline$€ 0-1000$ & $26(22.2)$ & $20(21.5)$ & $6(25.0)$ & n.s. \\
\hline$€ 1000-3000$ & $66(56.4)$ & $53(57.0)$ & $13(54.2)$ & \\
\hline$€ 3000-5000$ & $17(14.5)$ & $14(15.1)$ & $3(12.5)$ & \\
\hline$>€ 5000$ & $8(6.8)$ & $6(6.5)$ & $2(8.3)$ & \\
\hline Planned pregnancy & $77(65.8)$ & $63(67.7)$ & $14(58.3)$ & n.s. \\
\hline In vitro fertilization & $10(8.5)$ & $8(8.6)$ & $2(8.3)$ & \\
\hline \multicolumn{4}{|l|}{ Number of pregnancies } & n.s. \\
\hline First & $67(57.3)$ & $55(59.1)$ & $12(50.0)$ & \\
\hline Second & $37(31.6)$ & $27(29.0)$ & $10(41.7)$ & \\
\hline Third or more & $13(11.1)$ & $11(11.2)$ & $2(8.3)$ & \\
\hline \multicolumn{4}{|l|}{ Number of children } & n.s. \\
\hline None & $74(63.2)$ & $62(66.7)$ & $12(50.0)$ & \\
\hline One & $36(30.8)$ & $26(28.0)$ & $10(41.7)$ & \\
\hline Two & $7(6.0)$ & $5(5.4)$ & $2(8.3)$ & \\
\hline Gestational diabetes & $17(14.5)$ & $14(15.1)$ & $3(12.5)$ & n.s. \\
\hline
\end{tabular}


The majority was primiparous $(57.3 \%)$ with a mean gestational age of 32 weeks. Pregnancy was reportedly planned for $77(65.8 \%)$ of women of the sample, while 10 women $(8.5 \%)$ had become pregnant through in vitro fertilization. The prevalence of GDM was $14.5 \%$ (Table 1 ). From the initial sample of 117 women who participated in the prenatal data collection time point, 93 (79\%) were successfully reassessed in the first week postpartum follow-up. No significant demographic differences were found between these two groups (initial versus follow-up sample) (Table 1).

The median EPDS score during the third trimester of pregnancy was 5.00 (interquartile range $(\mathrm{IQR})=6.00)$ compared with $6.0(\mathrm{IQR}=7.0)$ in the first week postpartum. Using the validated cutoff of 12/13 for major depression, $12 \%$ had a likely diagnosis of depression during pregnancy and $15.1 \%$ in the first week postpartum (Table 2).

A significant association was found between presence of GDM and postpartum EPDS scores (Mann-Whitney $U=762.0, Z=2.25, P=0.024$ ). Women with GDM scored higher on the EPDS

Table 2 Descriptive statistics of prenatal and postnatal depressive symptoms

\begin{tabular}{lrrll}
\hline & $n$ & $\%$ & Median & IQR \\
\hline Pregnancy EPDS $(n=117)$ & & & 5.00 & 6.00 \\
$\quad$ Total score $\leqslant 12$ & 103 & 88.0 & & \\
$\quad$ Total score $\geqslant 13$ & 14 & 12.0 & & \\
Postpartum EPDS $(n=93)$ & & & 6.00 & 7.00 \\
$\quad$ Total score $\leqslant 12$ & 79 & 84.9 & & \\
$\quad$ Total score $\geqslant 13$ & 14 & 15.1 & & \\
\hline
\end{tabular}

IQR = interquartile range; EPDS = Edinburg Postnatal Depression Scale. within the first week postpartum (median $=8.00$, $\mathrm{IQR}=8.00)$ compared with women without GDM (median $=5.00, \mathrm{IQR}=7.00)$. No statistically significant relationship between GDM and prenatal EPDS scores was found (Mann-Whitney $U=974.5, Z=0.97, P=0.33$ ) (Table 3).

Similar results were reported with depression as a categorical variable (probable diagnosis $=$ EPDS score $\geqslant 13$ ). Significantly, more women with GDM screened positive for depression compared with non-GDM women (35.7 versus $11.4 \% ; \chi^{2}=5.501$, $P=0.02$ ). Moreover, women with GDM were significantly more likely to screen positive for PPD, even after controlling for confounding variables including age, monthly income, nulliparity, mode of delivery, and NICU admission. GDM was significantly associated with increased odds for PPD (adjusted odds ratio 4.69, 95\% CI 1.07-20.64).

\section{Discussion}

The aim of the present prospective study was to elucidate on the relationship between GDM and depressive symptoms during pregnancy and postpartum. Our findings revealed a significant association between GDM and depression symptoms in the postpartum period. Interestingly, no relationship was observed between GDM and depression scores in the third trimester of pregnancy.

Our results corroborate previous findings suggesting a link between GDM and PPD, but not antenatal depression. For instance, Kozhimannil et al. (2009) found that pre-pregnancy diabetes or GDM was independently associated with new onset of PPD. Similarly, Dalfrà et al. (2001) found

Table 3 Prenatal and postnatal depressive symptoms with health problems during pregnancy

\begin{tabular}{lccc}
\hline & No GDM & GDM & $P$-value \\
\hline EPDS pregnancy $(n=117)$ & & & \\
EPDS score [median (IQR)] & $6.00(7.0)$ & $7.00(5.5)$ & 0.33 \\
Probable depression [ $n(\%)]$ & $11(11)$ & $3(17.6)$ & 0.35 \\
EPDS postpartum $(n=93)$ & & & \\
EPDS score (median, IQR) & $5.00(7.0)$ & $8.00(8.0)$ & 0.02 \\
Probable depression ( $n, \%)$ & $9(11.4)$ & $5(35.7)$ & 0.02 \\
\hline
\end{tabular}

$\mathrm{GDM}=$ gestational diabetes mellitus; IQR =interquartile range; $E P D S=$ Edinburg Postnatal Depression Scale.

Probable depression diagnosis $=$ EPDS score $\geqslant 13$.

Primary Health Care Research \& Development 2017; 18: 441-447 
that women with type 1 diabetes mellitus and GDM had significantly higher levels of depressive symptoms at eight weeks postpartum compared with controls. More recently, Byrn and Penckofer (2015) found that women with GDM had higher rates of depression; however, their results were not statistically significant. These results were echoed by Katon et al. (2011) who found that neither pre-existing diabetes nor GDM was associated with increased risk of antenatal depression when adjusting for confounding factors.

Several studies, however, have failed to report a relationship between GDM and PPD. For instance, a large, prospective cohort study reported comparable rates of PPD symptoms among women with and without GDM (Kim et al., 2005). Another study conducted in Saudi Arabia by Al-Shahrani et al. (2011) using the same instrument and time intervals of assessments with our study, found no significant differences in PPD symptoms between women with and without GDM. Moreover, a recent study by Liu and Tronick (2013) showed that there was no association between PPD and higher rates of GDM after controlling for socio-demographic variables. A recent prospective study also failed to report a significant association between diabetes (including GDM and or pre-pregnancy diabetes) and PPD; however, women with pre-pregnancy diabetes were more likely to develop PPD compared with those without pre-pregnancy diabetes (Miller et al., 2015).

There are several possible explanations for the discrepant results among different studies. First, there are significant variations in the methodology and design, some studies are cross-sectional while others longitudinal, and there is considerable variation in postpartum time point assessment. Moreover, there are no unified diagnostic criteria for GDM and PPD, and thus it is reasonable to expect that the different ways of assessing the symptoms as well as the different time points of assessment can yield mixed findings. Furthermore, variability in sample size and heterogeneity in clinical as well as cultural characteristics of postpartum women may also play a role.

These discrepancies notwithstanding, there are possible mechanisms to support an association between PPD and GDM. Diabetes affects glycemic control and thyroid function, both of which impact the hypothalamic-pituitary-adrenal axis and cortisol levels (Lustman et al., 2000). These hormonal changes may be important contributing factors in the development of depression during the perinatal period (Chen et al., 2006; Kammerer et al., 2006). In addition, the stress of managing an illness that poses risks to the woman and the infant may exacerbate depressive symptoms in pregnant women and mothers (Séguin et al., 1995).

\section{Limitations}

Our study findings should be interpreted with caution due to some limitations. First, in our study we followed up women in the postpartum only once, a week after they gave birth. Including at least one additional assessment time point perhaps six months following birth would significantly improve the validity of EPDS scores. Second, the sample was relatively small to control for confounders, and during the postpartum reassessment $20.51 \%$ of the sample had dropped out. Despite these limitations, additional analysis found no significant differences between the initial and follow-up sample, suggesting that sample attrition followed a random pattern and was not due to systematic biases. In addition, we did not collect anthropometric data, such as prepregnancy body mass index; this is an important omission, given that obesity is a well-documented risk factor for GDM (Chu et al., 2007; Torloni et al., 2009) and a correlate of depression (Luppino et al., 2010) and perinatal depressive symptomatology (Molyneaux et al., 2014). Furthermore, our sample was recruited from a large urban center and the majority of the participating women had relatively high educational attainment, which may limit the generalizability of our findings to women with different demographic profiles.

\section{Future recommendations}

In view of these limitations, we hope that these preliminary results will stimulate further research to establish whether a link exists between PPD symptomatology and GDM. Further research should aim at assessing depression symptoms using structured diagnostic tools in addition to self-report measures, while GDM diagnosis should be based on biological screening rather than self-reports. Large representative samples will permit investigation of the relationship between GDM and perinatal 
depression adjusting for confounding factors. Assessment should be conducted at multiple time points, during first, second, and third trimester as well as at several time points postpartum (eg, one week, six month, one year).

\section{Conclusion}

Our study findings, albeit preliminary, may have important clinical implications. Women with GDM appear to be at risk for developing a mood disorder following childbirth. This finding points toward the need for healthcare providers to closely monitor women with GDM for PPD. There is compelling evidence for the deleterious effects of depression on the mother, the infant, and the entire family system (Murray et al., 1996; Weinberg and Tronick, 1998; Field, 2010). Thus, timely detection of PPD and identification of vulnerable groups to PPD are of great public health significance. Several screening measures for PPD have been developed, with sound psychometric properties and good acceptability and feasibility (El-Den et al., 2015).

In conclusion, the evidence from our study suggests that GDM is associated with depressive symptoms in the first week postpartum. Given the preliminary nature of the present study, our results should be replicated in larger, more robust studies.

\section{Acknowledgments}

The authors thank all the women who participated in this study.

\section{Authors' Contribution}

P.V. initiated the research, participated in study design and data analysis, conceived the study, designed and carried out the study, participated in data collection, and drafted the manuscript. A.C.S. participated in the study design, manuscript development, and data analysis and interpretation. Z.K. supervised statistical analysis and interpreted the data. E.V. participated in statistical analysis and manuscript preparation. M.M. participated in drafting of the manuscript and critical revision of the manuscript. I.M.Z. supervised the design of the study, data collection, and data analysis and interpretation. All authors read and approved the final manuscript.

Primary Health Care Research \& Development 2017; 18: 441-447

\section{Financial Support}

None.

\section{Conflicts of Interest}

None.

\section{References}

ACOG Committee on Practice Bulletins-Obstetrics 2004: ACOG practice bulletin \#55: management of postterm pregnancy. Obstetrics \& Gynecology 104, 639-46.

Al-Shahrani, M., Al-Sunaidi, M., Al-Amri, H., Al-Maswary, S. and Al-Gelban, K. 2011: Gestational diabetes and postpartum depression. The Arab Journal of Psychiatry 22, 133-37.

Bellamy, L., Casas, J.-P., Hingorani, A.D. and Williams, D. 2009: Type 2 diabetes mellitus after gestational diabetes: a systematic review and meta-analysis. The Lancet 373, 1773-779.

Bener, A., Saleh, N.M. and Al-Hamaq, A. 2011: Prevalence of gestational diabetes and associated maternal and neonatal complications in a fast-developing community: global comparisons. International Journal of Women's Health 3, 367-73.

Besser, A., Priel, B., Flett, G.L. and Wiznitzer, A. 2007: Linear and nonlinear models of vulnerability to depression: personality and postpartum depression in a high risk population. Individual Differences Research 5, 1-29.

Byrn, M. and Penckofer, S. 2015: Relationship between gestational diabetes and postpartum depression. Journal of Obstetric, Gynecologic, \& Neonatal Nursing 19, 246-55.

Chen, T.H., Lan, T.H., Yang, C.Y. and Juang, K.D. 2006: Postpartum mood disorders may be related to a decreased insulin level after delivery. Medical Hypotheses 66, 820-23.

Chu, S., Callaghan, W.M., Kim, S.Y., Schmid, C.H., Lau, J., England, L.J. and Dietz, P.M. 2007: Maternal obesity and risk of gestational diabetes mellitus. Diabetes Care 30, 2070-76.

Cox, J.L., Holden, J.M. and Sagovsky, R. 1987: Detection of postnatal depression: development of the 10-item Edinburgh Postnatal Depression Scale. British Journal of Psychiatry 150, 782-86.

Crowther, C.A., Hiller, J.E., Moss, J.R., McPhee, A.J., Jeffries, W.S. and Robinson, J.S. 2005: Effect of treatment of gestational diabetes mellitus on pregnancy outcomes. New England Journal of Medicine 352, 2477-486.

Dalfrà, M.G., Lapolla, A., Masin, M., Giglia, G., Dalla Barba, B., Toniato, R. and Fedele, D. 2001: Antepartum and early postpartum predictors of type 2 diabetes development in women with gestational diabetes mellitus. Diabetes \& Metabolism 27, 675-80.

Davé, S., Petersen, I., Sherr, L. and Nazareth, I. 2010: Incidence of maternal and paternal depression in primary care: a cohort study using a primary care database. Archives of Pediatrics \& Adolescent Medicine 164, 1038-44. 
de Groot, M., Anderson, R., Freedland, K.E., Clouse, R.E. and Lustman, P.J. 2001: Association of depression and diabetes complications: a meta-analysis. Psychosomatic Medicine 63, 619-30.

El-Den, S., O'Reilly, C.L. and Chen, T.F. 2015: A systematic review on the acceptability of perinatal depression screening. Journal of Affective Disorders 188, 284-303.

Evid Rep Technol Assess (Summ). 2005 Feb;(119):1-8.

Field, T. 2010: Postpartum depression effects on early interactions, parenting, and safety practices: a review. Infant Behavior and Development 33, 1-6.

Gaynes, B.N., Gavin, N., Meltzer-Brody, S., Lohr, K.N., Swinson, T., Gartlehner, G., Brody, S. and Miller, W.C. 2005: Perinatal depression: prevalence, screening accuracy, and screening outcomes. Evidence Report/Technology Assessment (Summary) 119, 1-8.

Golden, S.H., Lazo, M., Carnethon, M., Bertoni, A.G., Schreiner, P.J., Diez Roux, A.V., Lee, H.B. and Lyketsos, C. 2008: Examining a bidirectional association between depressive symptoms and diabetes. Journal of the American Medical Association 299, 2751-759.

Guariguata, L., Linnenkamp, U., Beagley, J., Whiting, D.R. and Cho, N.H. 2014: Global estimates of the prevalence of hyperglycaemia in pregnancy. Diabetes Research and Clinical Practice 103, 176-85.

Halkoaho, A., Pietilä, A.M., Huopio, H., Sintonen, H. and Heinonen, S. 2010: Does gestational diabetes affect women's health-related quality of life after delivery? European Journal of Obstetrics Gynecology and Reproductive Biology 148, 40-43.

International Association of Diabetes and Pregnancy Study Groups Consensus Panel, Metzger, B.E., Gabbe, S.G., Persson, B., Buchanan, T.A., Catalano, P.A., Damm, P., Dyer, A.R., Leiva, A.D., Hod, M., Kitzmiler, J.L., Lowe, L.P., McIntyre, H.D., Oats, J.J., Omori, Y., Schmidt, M.I. 2010: International association of diabetes and pregnancy study groups recommendations on the diagnosis and classification of hyperglycemia in pregnancy. Diabetes Care 33, 676-82.

Kammerer, M., Taylor, A. and Glover, V. 2006: The HPA axis and perinatal depression: a hypothesis. Archives of Women's Mental Health 9, 187-96.

Katon, J.G., Russo, J., Gavin, A.R., Melville, J.L. and Katon, W.J. 2011: Diabetes and depression in pregnancy: is there an association? Journal of Women's Health 20, 983-89.

Katon, W., Russo, J., Lin, E.H., Heckbert, S.R., Ciechanowski, P., Ludman, E.J. and Von Korff, M. 2009: Depression and diabetes: factors associated with major depression at five-year follow-up. Psychosomatics 50, 570-79.

Kim, C., Brawarsky, P., Jackson, R.A., Fuentes-Afflick, E. and Haas, J.S. 2005: Changes in health status experienced by women with gestational diabetes and pregnancy-induced hypertensive disorders. Journal of Women's Health 14, 729-36.

Kozhimannil, K.B., Pereira, M.A. and Harlow, B. 2009: Association between diabetes and perinatal depression among low-income mothers. Journal of the American Medical Association 301, 842-47.
Leonardoua, A.A., Zervas, Y.M., Papageorgiou, C.C., Marks, M.N., Tsartsara, E.C., Antsaklis, A., Christodoulou, G.N. and Soldatos, C.R. 2009: Validation of the Edinburgh Postnatal Depression Scale and prevalence of postnatal depression at two months postpartum in a sample of Greek mothers. Journal of Reproductive and Infant Psychology 27, 28-39.

Leung, B.M.Y. and Kaplan, B.J. 2009: Perinatal depression: prevalence, risks, and the nutrition link - a review of the literature. Journal of the American Dietetic Association 109, $1566-575$.

Liu, C.H. and Tronick, E. 2013: Rates and predictors of postpartum depression by race and ethnicity: results from the 2004 to 2007 New York City PRAMS survey (pregnancy risk assessment monitoring system). Maternal and Child Health Journal 17, 1599-610.

Luppino, F.S., de Wit, L.M., Bouvy, F., Stijnen, T., Cuijpers, P., Penninx, B.W. and Zitman, F.G. 2010: Overweight, obesity, and depression. Archives of General Psychiatry 67, 220-29.

Lustman, P.J., Anderson, R.J., Freedland, K.E., de Groot, M., Carney, R.M. and Clouse, R.E. 2000: Depression and poor glycemic control a meta-analytic review of the literature. Diabetes Care 23, 934-42.

Metzger, B.E. 2007: Long-term outcomes in mothers diagnosed with gestational diabetes mellitus and their offspring. Clinical Obstetrics and Gynecology 50, 972-79.

Miller, E.S., Peri, M.R. and Gossett, D.R. 2015: The association between diabetes and postpartum depression. Archives of Women's Mental Health 19, 183-86.

Mitanchez, D., Yzydorczyk, C. and Simeoni, U. 2015: What neonatal complications should the pediatrician be aware of in case of maternal gestational diabetes? World Journal of Diabetes 6, 734-43.

Molyneaux, E., Poston, L., Ashurst-Williams, S. and Howard, L.M. 2014: Obesity and mental disorders during pregnancy and postpartum: a systematic review and meta-analysis. Obstetrics and Gynecology 123, 857-67.

Murray, L., Fiori-Cowley, A., Hooper, R. and Cooper, P. 1996: The impact of postnatal depression and associated adversity on early mother-infant interactions and later infant outcome. Child Development 67, 2512-526.

Norhayati, M.N., Hazlina, N.H., Asrenee, A.R. and Emilin, W.M. 2015: Magnitude and risk factors for postpartum symptoms: a literature review. Journal of Affective Disorders $175,34-52$.

Séguin, L., Potvin, L., St-Denis, M. and Loiselle, J. 1995: Chronic stressors, social support, and depression during pregnancy. Obstetrics and Gynecology 85, 583-89.

Torloni, M.R., Betrán, A.P., Horta, B.L., Nakamura, M.U., Atallah, A.N., Moron, A.F. and Valente, O. 2009: Prepregnancy BMI and the risk of gestational diabetes: a systematic review of the literature with meta-analysis: Diagnostic in Obesity and Complications. Obesity Reviews 10, 194-203.

Weinberg, M.K. and Tronick, E.Z. 1998: The impact of maternal psychiatric illness on infant development. The Journal of Clinical Psychiatry 59 (Suppl 2), 53-61. 